\title{
Role of endoscopic transparent cap in managing pediatric foreign bodies in the entrance of the esophagus in a waking state
}

\author{
Yang Wang ${ }^{1}$, Wei Chen ${ }^{2}$ \\ ${ }^{1}$ Department of Gastroenterology, ${ }^{2}$ Department of Thoracic Surgery, The First People's Hospital of Taizhou, Taizhou 318020, China \\ Contributions: (I) Conception and design: Y Wang; (II) Administrative support: W Chen; (III) Provision of study materials or patients: All authors; \\ (IV) Collection and assembly of data: All authors; (V) Data analysis and interpretation: All authors; (VI) Manuscript writing: All authors; (VII) Final \\ approval of manuscript: All authors. \\ Correspondence to: Wei Chen, BA. Department of Thoracic Surgery, The First People's Hospital of Taizhou, No. 218 Hengjie, Taizhou 318020 , \\ China. Email: chenw021357@163.com.
}

\begin{abstract}
Background: This study aims to investigate the effect and safety of a gastroscope plus transparent cap in treating pediatric foreign bodies in the entrance of the esophagus in a waking state.

Methods: A total of 47 pediatric cases with foreign bodies were randomly divided into two groups: transparent cap group (24 cases), and the control group (23 cases). In the former group, one transparent cap was first installed on top of the gastroscope. Then, the foreign body was extracted using the gastroscope with a transparent cap in a waking state. In the other group, the foreign body was extracted using a gastroscope without a transparent cap in a waking state. Then, the time of extracting the foreign body, the success rate of extracting the foreign body and complication rate were compared between these two groups.

Results: The time of extraction in the transparent cap group was $6.78 \pm 1.76 \mathrm{~min}$, which was obviously shorter than that of the control group $(11.41 \pm 4.12 \mathrm{~min}, \mathrm{P}<0.05)$. In addition, the success rate of extraction in the former group $(23 / 24,95.8 \%)$ was higher than that of the latter group $(17 / 23,73.9 \%)(\mathrm{P}<0.05)$, and local mucosal bleeding correlated with the endoscopic operation in the former group was lower than that of the latter group $(\mathrm{P}<0.05)$.

Conclusions: The use of a transparent cap could shorten the time of extraction and improve the success rate of extraction through a gastroscope in treating pediatric foreign bodies in the entrance of the esophagus in a waking state. This is an effective and safe treatment approach for pediatric foreign bodies in the entrance of the esophagus.
\end{abstract}

Keywords: Transparent cap; waking state; pediatric; foreign body in the entrance of esophagus

Submitted Feb 25, 2019. Accepted for publication Jul 09, 2019.

doi: $10.21037 /$ jtd.2019.09.39

View this article at: http://dx.doi.org/10.21037/jtd.2019.09.39

\section{Introduction}

Children often develop esophageal foreign bodies that are easily incarcerated at the entrance of the esophagus. The main type of foreign body is coins, accounting for $57.2-88 \%$ (1-4). Foreign bodies also include buttons, batteries, fishbone, and other sharp or pointed foreign bodies (5). Once a foreign body is incarcerated at the esophagus of a child, the child may present with crying after eating, vomiting, salivation and dysphagia (6). Foreign bodies should be removed in time. If these foreign bodies are not removed in time, severe patients can be complicated with issues such as esophageal fistula, perforation, mediastinal abscess $(7,8)$. If the large vessels around the esophagus are affected, fatal bleeding may even occur. Due to small age, children are unable to cooperate with gastroscopy compared to adults in the waking state. The small space at the entrance of esophagus affects the success rate of foreign body removal, prolongs the operation time of foreign body removal, and easily induces complications. At present, there 
Table 1 General data of children in the two groups

\begin{tabular}{lcc}
\hline Groups & Transparent cap group & Control group \\
\hline Cases & 24 & 23 \\
Sex (male/female) & $15 / 9$ & $15 / 8$ \\
Age (months) & $65.08 \pm 23.32$ & $69.30 \pm 21.92$ \\
Foreign body distribution (cases) & \\
Coin & 16 & 15 \\
Ornaments & 2 & 3 \\
fishbone & 1 & 0 \\
Tree branch & 0 & 1 \\
Animal bone & 2 & 0 \\
Jujube core & 1 & 0 \\
Button & 1 & 2 \\
Button battery & 1 & 1 \\
Paper clip & 0 & 1 \\
\hline & &
\end{tabular}

are few reports of large sample randomized controlled studies on the diagnosis and treatment of esophageal foreign bodies with digestive endoscopy in children both locally and internationally (9). However, there is a lack of guidelines for the diagnosis and treatment of esophageal foreign bodies in children. From January 2012 to June 2018, 47 children with esophageal foreign bodies were treated using a gastroscope in our hospital. The present study aims to seek for a safe and effective endoscopic treatment approach for esophageal foreign bodies in children. The details are reported as follows.

\section{Methods}

\section{General information}

A total of 47 children were enrolled in the present study. Before the gastroscopy, neck computed tomography (CT) was performed to check the foreign body at the entrance of the esophagus, and the contraindications of gastroscopy for esophageal perforation were excluded. According to the random number table method, these 47 children were divided into two groups: transparent cap group and control group. The transparent cap group comprised 24 patients, including 15 male patients and nine female patients. The age of these patients ranged from 2 to 11 years old, with an average age of $65.08 \pm 23.32$ months old. Foreign body distribution: coins were found in 16 patients, ornaments were found in two patients, a fishbone was found in one patient, animal bones were found in two patients, a jujube core was found in one patient, a button battery was found in one patient, and a button was found in one patient. The control group comprised of 23 patients, including 15 male patients and eight female patients. The age of these patients ranged from 19 months old to 11 years old, with an average age of $69.30 \pm 21.92$ months old. Foreign body distribution: coins were found in 15 patients, ornaments were found in 3 patients, branches were found in one patient, a button battery was found in one patient, and a paper clip was found in one patient. The difference in composition of foreign bodies between these two groups was not statistically significant. No chronic or hemorrhagic diseases were found in patients in the present study (Table 1). This study was conducted in accordance with the declaration of Helsinki. This study was conducted with approval from the Ethics Committee of Affiliated Huangyan Hospital of Wenzhou Medical University. Written informed consent was obtained from the participants.

\section{Instruments}

The instruments used in the present study included an Olympus 260 electronic gastroscope, alligator forceps, trielcon, biopsy forceps, snares, transparent caps and oral retainers. Appropriate instruments were selected according to the situation of the foreign body during the operation.

\section{Methods and steps}

(I) Relevant preoperative inspections were thoroughly performed, such as CT of the neck, chest and abdomen, in order to determine the nature, location and quantity of foreign bodies, exclude the presence of gastrointestinal perforation, especially esophageal perforation, and prevent the gastroscopy from being influenced by esophageal barium meal examination. In addition, routine blood test and coagulation function examination were also performed to exclude low platelet count and coagulation dysfunction;

(II) Fasting and fluid deprivation before the operation: before the operation, children underwent fasting for six hours and fluid deprivation for four hours, which could reduce the occurrence of complications, such as vomiting and asphyxia 
during the operation;

(III) Medication before the operation: according to the weight of these children, children orally used appropriate amounts of lidocaine mucilage for local pharyngeal anesthesia before the operation. Since all children in the present study underwent emergency gastroscopy, intravenous anesthesia was not performed to avoid vomiting and asphyxia;

(IV) Condition communication: doctors communicated with the parents and children about the condition. If the child was older, the doctor communicated with the child, and the child's cooperation was obtained. If the child was younger, family members should assist and cooperate during the gastroscopy. If the child was really unable to cooperate, the operation under the waking state would be given up, and the child would undergo gastroscopic foreign body removal under general anesthesia with endotracheal intubation;

(V) Methods for foreign body removal: the transparent cap group-a transparent cap was installed at the front end of the gastroscope, making the front end of the transparent cap protrude from the gastroscope by approximately $1 \mathrm{~cm}$. Under the direct vision of the gastroscope, different foreign body removal instruments were selected according to the situation of the foreign body, and the foreign body was removed. The control group: A gastroscope was directly inserted to search for the foreign body, foreign body removal instruments were used for foreign body removal, and the operation time was recorded;

(VI) Postoperative examination: after removing the foreign bodies, the gastroscope was inserted again to check whether there were complications, such as breakage, erosion, bleeding and perforation of the esophageal mucosa. If there was bleeding in the esophageal mucosa, endoscopic hemostasis was performed. If there was perforation of the esophagus, the child was fasted and underwent gastrointestinal decompression, and a consultation in the Department of Thoracic Surgery was performed to determine whether a surgical treatment was needed (Figure 1). The procedures were performed by the same team of endoscopists.

\section{Statistical analysis}

Data were statistically analyzed using SPSS 10 software.
Measurement data in the experiment were expressed as mean \pm standard deviation $\left(X_{ \pm} \mathrm{SD}\right)$, and compared between two groups using $t$-test. Count data were compared using Chi-square test. $\mathrm{P}<0.05$ was considered statistically significant.

\section{Results}

Transparent cap group: there were 24 foreign bodies in 24 children, and a total of 23 foreign bodies in 23 children were successfully removed. The success rate was $95.8 \%$, and the average time for foreign body removal was $6.78 \pm 1.76 \mathrm{~min}$. Among these 24 children, one child could not tolerate the gastroscopy under the waking state, and was treated with a transparent cap combined with gastroscopy to remove the foreign body after general anesthesia with endotracheal intubation. Control group: There were 23 foreign bodies in 23 children, and a total of 17 foreign bodies in 17 children were successfully removed. The success rate of the foreign body removal was $73.9 \%$, and the average time for the foreign body removal was $11.41 \pm 4.12 \mathrm{~min}$. Among these 23 children, two children could not tolerate the gastroscopy under the waking state, and were treated with a transparent cap combined with gastroscopy to remove the foreign bodies after general anesthesia with endotracheal intubation. In addition, the foreign bodies of four children were difficult to remove due to poor visual field in the gastroscope, and gastroscopy combined with a transparent cap was used instead, allowing these foreign bodies to be successfully removed. The time for foreign body removal was shorter in the transparent cap group than in the control group $(\mathrm{P}<0.05)$, and the success rate of foreign body removal was higher in the transparent cap group than in the control group $(\mathrm{P}<0.05)$ (Table 2$)$.

In the transparent cap group, four children had mucosal damage caused by foreign bodies and a few bleedings, but no complications, such as obvious massive hemorrhage, esophageal perforation, vomiting and foreign body-induced asphyxia, were found. In the control group, three children had mucosal damage caused by foreign bodies and a few bleedings. In addition, four children in the control group had local mucosal damage and bleedings caused by the crocodile forceps due to poor visual field. The bleedings were stopped after local spraying of noradrenaline. No complications, such as obvious massive hemorrhage, esophageal perforation, vomiting and foreign body-induced asphyxia, were found. The difference in local mucosal bleeding correlated with the endoscopic operation between 

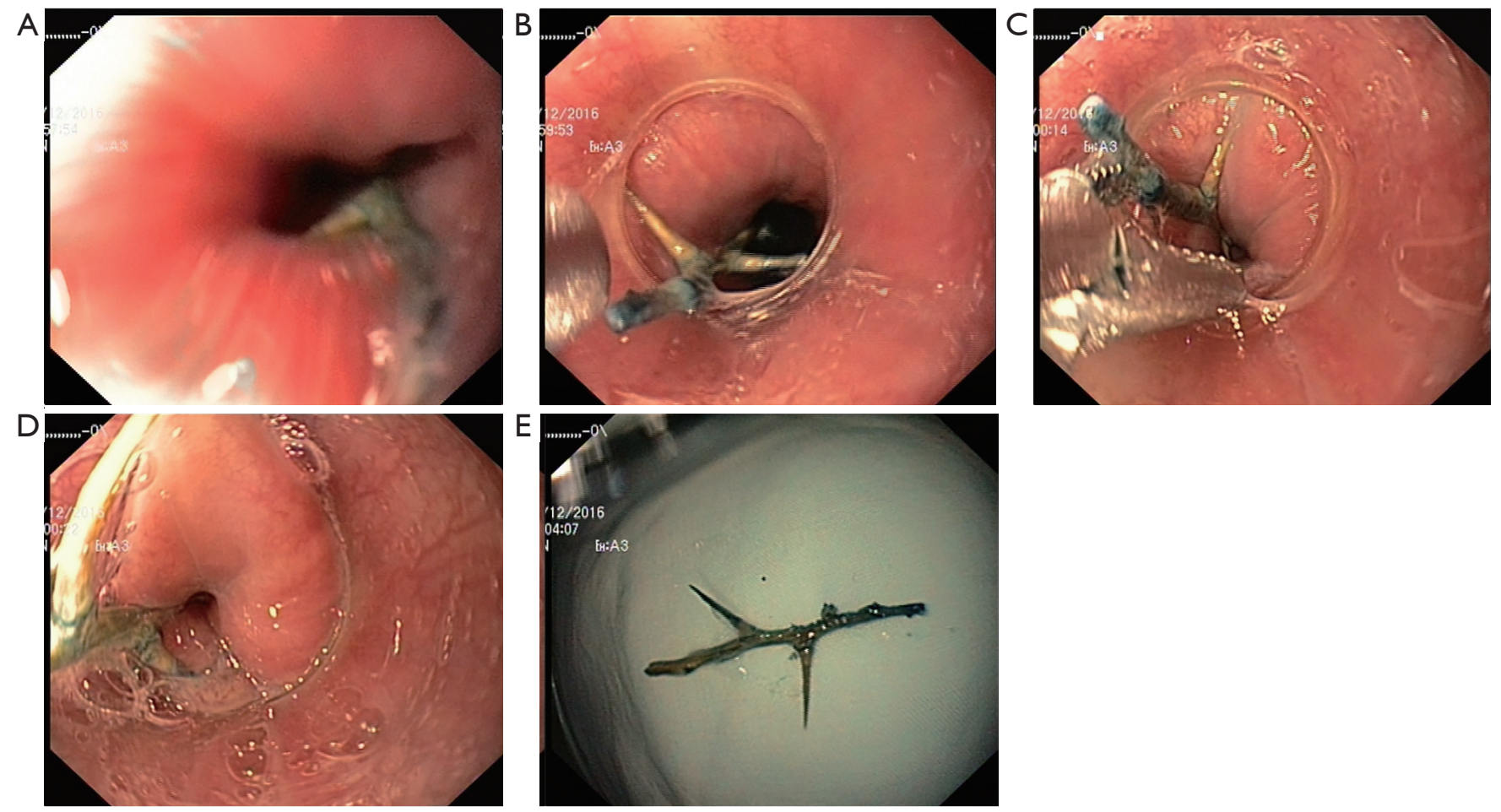

Figure 1 The operation process of gastroscopy with transparent caps to remove foreign bodies in children's esophagus under the waking state: (A) the space of the lumen at the entrance of the esophagus is narrow and small, and dendritic foreign bodies could be observed; (B) the transparent cap was installed at the front end of the gastroscope, the lumen space and endoscopic visual field increased, and a shallow foreign body penetrating the esophageal wall by one end could be seen; (C) crocodile forceps was inserted into the biopsy forceps, and the foreign body was clamped; (D) the foreign body was clamped with crocodile forceps and pulled into a transparent cap; (E) the dendritic foreign body is removed, which was a sharp foreign body.

Table 2 Comparison of methods for foreign body removal in the two groups

\begin{tabular}{|c|c|c|c|c|}
\hline Groups & Cases & $\begin{array}{l}\text { The average time for the } \\
\text { foreign body removal (min) }\end{array}$ & Success (cases) & $\begin{array}{l}\text { Local mucosal bleeding correlated } \\
\text { with the endoscopic operation (cases) }\end{array}$ \\
\hline Transparent cap group & 24 & $6.78 \pm 1.76$ & 23 & 0 \\
\hline Control group & 23 & $11.41 \pm 4.12$ & 17 & 4 \\
\hline
\end{tabular}

these two groups was statistically significant $(\mathrm{P}<0.05)$.

\section{Discussion}

Esophageal foreign bodies in children are common clinical emergencies in pediatrics. At present, there is no statistical data on its incidence. In our hospital, esophageal foreign bodies account for approximately $0.2 \%$ of the causes of gastroscopy. There are three physiological stenoses in the esophagus, and the diameter of the esophagus in children is relatively small, making it more prone to foreign body incarceration, especially in the upper esophagus. Furthermore, approximately $68-73 \%$ of esophageal foreign bodies in children are incarcerated at the entrance of the esophagus $(2,4,10)$, which is physiological stenosis. In adults, approximately $80 \%$ of esophageal foreign bodies can be spontaneously discharged, but $10-20 \%$ of foreign bodies cannot be expelled from the body, in which endoscopic or surgical procedures are needed $(11,12)$. Since children have smaller esophageal diameters, they are more prone to foreign body incarceration. A study revealed that merely approximately $40 \%$ of foreign bodies in 
children can be spontaneously discharged (13), and greater number esophageal foreign body cases in children requires endoscopic or surgical procedures.

With the rapid development of endoscopy and endoscopic accessories, gastroscopy has the advantages of high success rate of foreign body removal, high comfort degree, and less complications $(14,15)$. Therefore, the application of gastroscopy has become more and more extensive in the treatment of esophageal foreign bodies. At present, endoscopic accessories, such as snares, fetching basket, rat tooth forceps, trielcon and fetching balloon, have been routinely used in clinical gastroscopic foreign body removal $(11,16,17)$. Most esophageal foreign bodies need emergency treatment, in order to avoid complications, such as vomiting, asphyxia, and accidental aspiration of foreign bodies into the airway during the gastroscopy, and most esophageal foreign bodies are operated under gastroscopy in the waking state. Patients who are unable to cooperate during the gastroscopy can undergo endotracheal intubation gastroscopic removal under general anesthesia. However, in the waking state, nausea will be more obvious in children, which further narrows the narrow space in the entrance of the esophagus, and limit the operation space for the gastroscopy. Furthermore, the endoscopic visual field cannot be fully exposed, the operation difficulty is increased, the operation time is prolonged, and the success rate of foreign body removal is decreased (18). At present, few studies at home and abroad have reported that the transparent cap has advantages in the treatment of esophageal foreign bodies (18-20). By carefully studying these reports, two limitations were revealed: first, there was no detailed classification of anesthetic state or waking state in these studies, and there are great differences in the responses of patients to throat stimulation when gastroscopy was performed in these two different states; second, these were all studies on foreign bodies at the entrance of the esophagus in adults or in adults with very few children, and there were also significant differences in compliance and the coordination of gastroscopy between children and adults, as well as in the anatomical spatial size of the entrance of the esophagus. These factors have great impact on the success rate of foreign body removal and the incidence of complications. Therefore, in the past six years, our hospital attempted to remove foreign bodies at the entrance of the esophagus in children by gastroscopy combined with a transparent cap in the waking state, and relatively satisfactory curative effects were achieved.

In the present study, foreign bodies in 23 children in the transparent cap group were successfully removed, and the success rate was $95.8 \%$, which was significantly higher than that in the control group (73.9\%). Furthermore, the average time for removal of foreign bodies was $6.78 \pm 1.76 \mathrm{~min}$ in the transparent cap group, which was significantly shorter than that in the control group $(11.41 \pm 4.12 \mathrm{~min})$, suggesting that the use of a transparent cap can significantly improve the success rate of foreign body removal and shorten the time for foreign body removal. In addition, the rate of esophageal mucosa damage was lower in the transparent cap group than in the control group, suggesting that the use of transparent caps can reduce complications associated with gastroscopy. In the present study, in the transparent cap group, a transparent cap was equipped at the head end of the gastroscope, making the head end of the transparent cap protrude out of the head end of the gastroscope by $1 \mathrm{~cm}$. On the basis of reviewing previous literatures (18-23), this approach was considered to have the following advantages: (I) the setting of a transparent cap at the head end of the gastroscope stretches the esophageal lumen, increases the operation space and operation visual field, improves the stability of the front end of the gastroscope, decreases the repeated endoscopic insertions caused by endoscopic slippage, helps in the discovery of foreign bodies, and prevents the front end of endoscopy from exceeding the lesions in the process of inserting the endoscope, thereby reducing missed diagnosis and increasing the success rate of foreign body removal; (II) the use of a transparent cap increases the space of the endoscopic operation, which can allow the biopsy forceps and crocodile forceps to be opened freely in the transparent cap, and avoid clamping the esophageal mucosa when clamping the foreign bodies; (III) some small sharp foreign bodies are pulled into the transparent cap, which reduces the iatrogenic injury and perforation caused by sharp foreign bodies piercing into the esophagus during the removal process, and better fixes the foreign bodies to avoid the falling of foreign bodies when the gastroscope is withdrawn to the piriform recess, or the occurrence of secondary foreign bodies accidentally falling off the gastroscope and going into the trachea; (IV) the transparent cap is moved from right to left to stretch the mucosa of the esophageal wall, and allow some shallow foreign bodies piercing into the esophageal wall to loosen, facilitating the removal by forceps, and avoiding complications, such as perforation caused by foreign bodies penetrating into the esophageal wall when clamped.

At present, some studies have reported that it is a safe and effective method to successfully remove foreign bodies in 
the digestive tract by gastroscopy under general anesthesia with endotracheal intubation. Our hospital has attempted this method, and encountered the following problems: (I) after endotracheal intubation and balloon inflation, the anatomical structure of the entrance of the esophagus changed, which narrowed the space at the entrance of the esophagus, and increased operation difficulty; (II) in some patients with foreign bodies at the entrance of the esophagus, especially those the foreign bodies piercing into the esophageal wall in the sagittal pattern at both ends, iatrogenic secondary injuries, such as esophageal injury exacerbation or even esophageal perforation easily occurred after balloon inflation and endotracheal intubation; (III) patients who received general anesthesia after endotracheal intubation require hospitalization, which increases the patient's medical expenses; (IV) After endotracheal intubation, iatrogenic changes in the protective barrier of the trachea occur, which increases the incidence of respiratory tract infection; (V) in the course of anesthesia, especially in the interval between endotracheal intubation general induction anesthesia, when muscle relaxation occurs, vomiting, asphyxia and foreign body falling into the trachea can easily occur. Since the present study is on foreign bodies at the esophageal entrance of children, the above-mentioned problems easily occur after anesthesia with endotracheal intubation, especially for children under 1-2 years old. The entrance of the esophagus was relatively narrow, and the space at the entrance of the esophagus was further narrowed after endotracheal intubation, which affected the insertion and subsequent operation of the gastroscope to a large extent. Hence, in the present study, it was advocated that the gastroscopic removal of foreign bodies at the entrance of the esophagus in children should be performed under the waking state.

In the present study, gastroscopy combined with a transparent cap could significantly shorten the time for foreign body removal, improve the success rate of foreign body removal, and reduce the incidence of complications associated with gastroscopy, which is a simple, safe and effective method for removing foreign bodies. At present, some viewpoints also suggest that the following: after the transparent cap was installed at the front end of the gastroscope, the discomfort of patients during the examination was increased, and the difficulty of esophageal foreign body operation in children was increased. Hence, a soft and deformable transparent cap can be used to reduce the discomfort at insertion. Clinical workers also need to continuously improve their operation skills, in order to reduce the sufferings of these patients and improve the compliance of patients. Intravenous anesthesia can be performed to relieve the pain of patients when necessary.

\section{Acknowledgments}

None.

\section{Footnote}

Conflicts of Interest: The authors have no conflicts of interest to declare.

Ethical Statement: The authors are accountable for all aspects of the work in ensuring that questions related to the accuracy or integrity of any part of the work are appropriately investigated and resolved. This study was conducted with approval from the Ethics Committee of the First People's Hospital of Taizhou. Written informed consent was obtained from the participants.

\section{References}

1. Hong KH, Kim YJ, Kim JH, et al. Risk factors for complications associated with upper gastrointestinal foreign bodies. World J Gastroenterol 2015;21:8125-31.

2. Denney W, Ahmad N, Dillard B, et al. Children will eat the strangest things: a 10-year retrospective analysis of foreign body and caustic ingestions from a single academic center. Pediatr Emerg Care 2012;28:731-4.

3. Zhou LQ, Zhao H, Peng KR, et al. Endoscopic management of ingested foreign bodies in the upper gastrointestinal tract in childhood: a retrospective study of 1334 cases. Zhonghua Er Ke Za Zhi 2018;56:495-9.

4. Little DC, Shah SR, St Peter SD, et al. Esophageal foreign bodies in the pediatric population: our first 500 cases. J Pediatr Surg 2006;41:914-8.

5. Lee JH. Foreign Body Ingestion in Children. Clin Endosc 2018;51:129-36.

6. Heinzerling NP, Christensen MA, Swedler R, et al. Safe and effective management of esophageal coins in children with bougienage. Surgery 2015;158:1065-70; discussion 1071-2.

7. Darwish HS, Qamar SR. Pediatric foreign body ingestion and esophageal impaction. Saudi Med J 2016;37:1276-8.

8. Magalhães-Costa P, Carvalho L, Rodrigues JP, et al. Endoscopic Management of Foreign Bodies in the Upper Gastrointestinal Tract: An Evidence-Based Review Article. 
GE Port J Gastroenterol 2015;23:142-52.

9. Kramer RE, Lerner DG, Lin T, et al. Management of ingested foreign bodies in children: a clinical report of the NASPGHAN Endoscopy Committee. J Pediatr Gastroenterol Nutr 2015;60:562-74.

10. Kay M, Wyllie R. Pediatric foreign bodies and their management. Curr Gastroenterol Rep 2005;7:212-8.

11. ASGE Standards of Practice Committee, Ikenberry SO, Jue TL, et al. Management of ingested foreign bodies and food impactions. Gastrointest Endosc 2011;73:1085-91.

12. Birk M, Bauerfeind P, Deprez PH, et al. Removal of foreign bodies in the upper gastrointestinal tract in adults: European Society of Gastrointestinal Endoscopy (ESGE) Clinical Guideline. Endoscopy 2016;48:489-96.

13. Rybojad B, Niedzielska G, Niedzielski A, et al. Esophageal foreign bodies in pediatric patients: a thirteen-year retrospective study. ScientificWorldJournal 2012;2012:102642.

14. Yan XE, Zhou LY, Lin SR, et al. Therapeutic effect of esophageal foreign body extraction management: flexible versus rigid endoscopy in 216 adults of Beijing. Med Sci Monit 2014;20:2054-60.

15. Gmeiner D, von Rahden BH, Meco C, et al. Flexible versus rigid endoscopy for treatment of foreign body impaction in the esophagus. Surg Endosc 2007;21:2026-9.

16. Asge Technology Committee, Diehl DL, Adler DG, et

Cite this article as: Wang Y, Chen W. Role of endoscopic transparent cap in managing pediatric foreign bodies in the entrance of the esophagus in a waking state. J Thorac Dis 2019;11(10):4357-4363. doi: 10.21037/jtd.2019.09.39 al. Endoscopic retrieval devices. Gastrointest Endosc 2009;69:997-1003.

17. Chauvin A, Viala J, Marteau P, et al. Management and endoscopic techniques for digestive foreign body and food bolus impaction. Dig Liver Dis 2013;45:529-42.

18. Zhang S, Wang J, Wang J, et al. Transparent cap-assisted endoscopic management of foreign bodies in the upper esophagus: a randomized, controlled trial. J Gastroenterol Hepatol 2013;28:1339-42.

19. Zhang S, Cui Y, Gong X, et al. Endoscopic management of foreign bodies in the upper gastrointestinal tract in South China: a retrospective study of 561 cases. Dig Dis Sci 2010;55:1305-12.

20. Patel NC, Fry LC, Mönkemüller KE. Modified suction cap technique for endoscopic management of esophageal food impaction. Endoscopy 2003;35:548.

21. Zhang Y, Xu XL, Li HJ, et al. Study on the Role of Transparent Cap in the Treatment of Small Foreign Bodies in the Esophagus. Mod Diagn Treat 2017;28:998-9.

22. Yan JY, Lei PG, Li QL, et al. Effect of gastroscopic lucency cap on extracting small foreign body in the entrance of esophagus. Chin J Gastroenterol Hepatol 2011;20:435-6.

23. Yang GY, Guo XH, Gong FY, et al. A control study of the upper esophageal foreign bodies removal with endoscopic caps. Chin J Gastroenterol Hepatol 2013;22:645-7. 